\title{
Regulation of soluble CD127 protein release and corresponding transcripts expression in T lymphocytes from septic shock patients
}

\author{
Julie Mouillaux ${ }^{1,2+}$, Camille Allam ${ }^{1,3+}$, Thomas Rimmelé ${ }^{1,4}$, Thomas Uberti ${ }^{4}$, Benjamin Delwarde ${ }^{4}$, Julien Textoris ${ }^{1,2,4}$, \\ Guillaume Monneret ${ }^{1,2,3}$, Estelle Peronnet ${ }^{1,2^{*+}}$ (D) and Fabienne Venet ${ }^{1,2,3+}$
}

\author{
* Correspondence: \\ estelle.peronnet@biomerieux.com \\ †ulie Mouillaux, Camille Allam, \\ Estelle Peronnet and Fabienne \\ Venet contributed equally to this \\ work. \\ ${ }^{1}$ EA 7426 «Pathophysiology of \\ injury-induced immunosuppression \\ (PI3) » Lyon 1 University / Hospices \\ Civils de Lyon / bioMérieux, 5 place \\ d'Arsonval, 69437 Lyon Cedex 03, \\ France \\ ${ }^{2}$ Joint Research Unit \\ HCL-bioMérieux-Lyon 1 University, \\ Hôpital Edouard Herriot, 5 place \\ d'Arsonval, 69003 Lyon, France \\ Full list of author information is \\ available at the end of the article
}

To the Editor,

Sepsis is the leading cause of death for critically ill patients. Septic patients develop $\mathrm{T}$ lymphocyte dysfunctions associated with increased mortality and nosocomial infections [1]. Therefore, IL-7 has been recently evaluated in a clinical trial to reverse these alterations [2]. IL-7 receptor (IL-7R) is composed of an IL-7-specific chain (CD127) and a common receptor $\gamma$-chain [3]. IL-7R exists in a soluble form (sCD127) [4], resulting from shedding from the cell surface or transcriptional regulation $[5,6]$. Several transcripts missing the exon 6, coding for the transmembrane domain have been identified, such as IL7R3 $[4,6]$ and IL7R7 (Ensembl). We recently showed that plasmatic sCD127 concentration and whole blood IL7R3 transcript expression were decreased in septic shock $[7,8]$. However, $\mathrm{sCD} 127$ protein and transcripts regulations in survivor and non-survivor patients differed, with higher SCD127 concentration and lower IL7R3 expression in non-survivors. Therefore, the regulation of soluble IL-7R in sepsis remains not fully understood.

In this study, we evaluated sCD127 release and corresponding transcripts IL7R3 and IL7R7 expressions in purified T cells from septic shock patients. After approval by local ethics committee, $\mathrm{T}$ cells were isolated from 32 septic shock patients (Table 1) and 31 healthy volunteers (HV) (Additional file 1).

Interestingly, the sCD127 release tended to be higher in patients' $\mathrm{T}$ cells supernatant compared to HV (Fig. 1). This contrasts with the decreased plasmatic sCD127 concentration in septic shock patients [7], possibly impacted by lymphopenia. Both IL7R3 and IL7R7 transcripts expressions were decreased in septic shock patients' T cells (Fig. 2), as we previously observed in whole blood [8]. While whole blood IL7R3 and IL7R7 expressions may be impacted by sepsis-induced lymphopenia, these transcripts are also intrinsically regulated in $\mathrm{T}$ cells.

To delineate soluble IL-7R regulation in sepsis, we aimed to reproduce ex vivo its expression pattern observed in patients, by stimulating T cells from HV with IL-7 or TCR activation, known to regulate CD127 expression [9]. sCD127 was spontaneously 
Table 1 Clinical characteristics of septic shock patients

\begin{tabular}{|c|c|}
\hline Parameters & Septic shock patients $(n=32)$ \\
\hline Sex, male & $26(68 \%)$ \\
\hline Age, years & 70 [62-76] \\
\hline SAPS II score & $61[51-82]$ \\
\hline SOFA score $(n=31)$ & $9[8-12]$ \\
\hline \multicolumn{2}{|c|}{ Charlson co-morbidity score $(n=30)$} \\
\hline 0 & $3(10 \%)$ \\
\hline 1 & $14(47 \%)$ \\
\hline$>1$ & $13(43 \%)$ \\
\hline \multicolumn{2}{|l|}{ Mac Cabe score $(n=31)$} \\
\hline Non-fatal diseases & $21(68 \%)$ \\
\hline Ultimately fatal diseases & $9(29 \%)$ \\
\hline Rapidly fatal diseases & $1(3 \%)$ \\
\hline \multicolumn{2}{|l|}{ Initial infection } \\
\hline Abdominal infection & $16(50 \%)$ \\
\hline Pneumopathy & $4(12.5 \%)$ \\
\hline Urinary infection & $4(12.5 \%)$ \\
\hline Other & $8(25 \%)$ \\
\hline \multicolumn{2}{|l|}{ Type of admission } \\
\hline Medical & $13(41 \%)$ \\
\hline Emergency surgery & $18(56 \%)$ \\
\hline Elective surgery & $1(3 \%)$ \\
\hline \multicolumn{2}{|c|}{ Microbiological documentation } \\
\hline Gram negative & $10(31 \%)$ \\
\hline Gram positive & $7(22 \%)$ \\
\hline Other & $1(3 \%)$ \\
\hline Non-documented & $14(44 \%)$ \\
\hline Mortality at D28 & $9(28 \%)$ \\
\hline Lactate at D1 (mmol/L) & $2.8[1.7-5.6]$ \\
\hline Lymphocytes at D3 $\left(10^{9} / \mathrm{L}\right)$ & $1.05[0.6-1.35]$ \\
\hline $\mathrm{mHLA}-\mathrm{DR}$ at $\mathrm{D} 3(\mathrm{AB} / \mathrm{C})$ & $8199[3076-12,171]$ \\
\hline
\end{tabular}

For clinical parameters, categorial data are presented as numbers of cases and percentages of the total population in brackets. Continuous data and biological parameters are presented as medians and interquartile ranges [Q1-Q3]. Simplified Acute Physiology Score II (SAPS II) and Mac Cabe score were calculated on admission. Sequential Organ Failure Assessment (SOFA) score was measured after $24 \mathrm{~h}$ of intensive care unit stay. mHLA-DR (AB/C): number of anti-HLA-DR antibodies bound per monocyte

released from non-stimulated T cells (Fig. 3a). During IL-7 stimulation, sCD127 protein release and transcripts expression decreased. In contrast, TCR activation induced an opposite regulation of sCD127 and corresponding transcripts (Fig. 3b): sCD127 concentration increased, as previously described [10], while IL7R7 and IL7R3 transcripts expressions decreased. This suggests that these transcripts are not the main source of sCD127 in this context, and that other mechanisms, such as shedding of membrane CD127, might occur. Overall, ex vivo TCR activation partly reproduced soluble IL-7R expression pattern observed in septic shock, suggesting that the initial $\mathrm{T}$ cell activation shown to occur in sepsis $[11,12]$ could participate to soluble IL-7R regulation, both at the transcriptional and protein levels. 


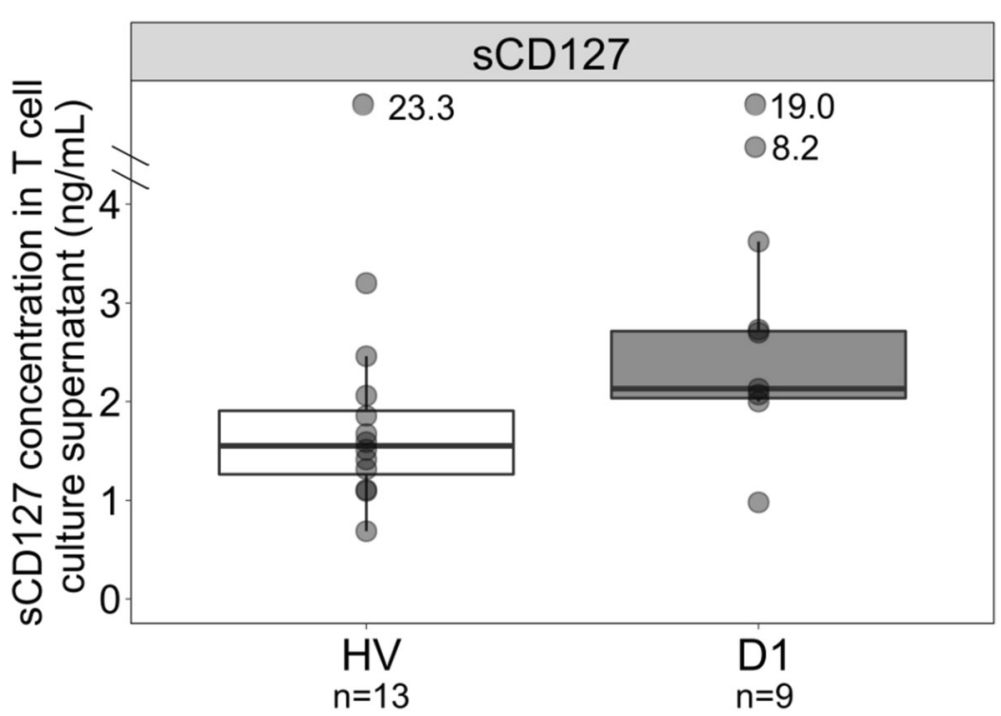

Fig. 1 sCD127 protein release from purified T cells from septic shock patients. sCD127 release was quantified in supernatants of purified T cells of septic shock patients $(D 1, n=9)$ in comparison with healthy volunteers ( $H V, n=13)$ after $48 \mathrm{~h}$ of culture without any stimulation. Data are presented as Tukey boxplots. Mann-Whitney tests were used to compare values between septic shock patients and HV. ${ }^{* *} p<0.01,{ }^{* *} p<0.001$. See Additional file 1 for details
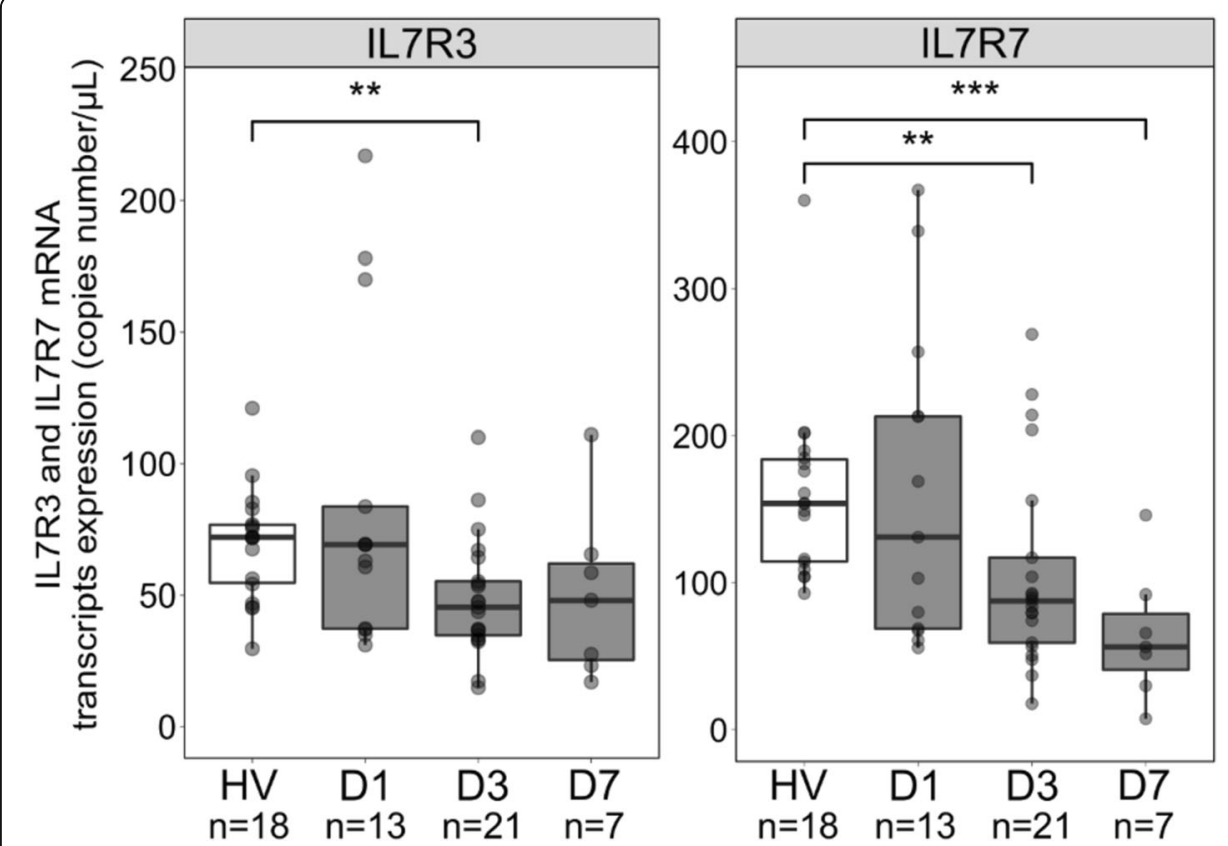

Fig. 2 IL7R mRNA transcripts expression in purified T cells from septic shock patients. Gene expressions of the IL7R3 and IL7R7 transcripts were measured using RT-qPCR from RNA from purified T cells from septic shock patients at D1 $(n=13)$, D3 $(n=21)$, and D7 $(n=7)$ in comparison with HV $(n=18)$. Data are presented as Tukey boxplots. Mann-Whitney tests were used to compare values between septic shock patients and HV. ${ }^{* *} p<0.01,{ }^{* *} p<0.001$. See Additional file 1 for details 

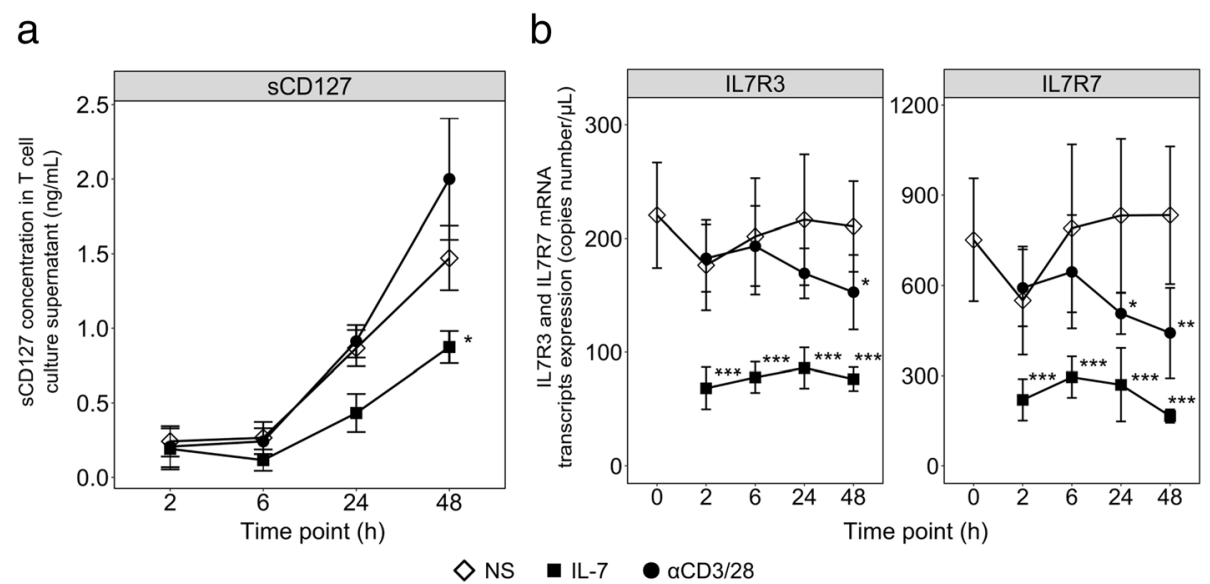

Fig. 3 sCD127 release and transcripts expression upon ex vivo stimulation of healthy volunteers' purified T cells. a SCD127 protein release and $\mathbf{b}$ IL7R3 and IL7R7 mRNA transcripts expressions were measured in purified T cells from healthy volunteers (HV) stimulated ex vivo after the indicated time of incubation with $\mathrm{L}-7(n=5,10 \mathrm{ng} / \mathrm{mL})$, anti-CD3/CD28 antibodies coated beads ( $n=5, \mathrm{aCD} 3 / 28,1: 2$ bead to cells ratio), or not stimulated ( $n=10, \mathrm{NS})$. For each $\mathrm{HV}$ and each condition of stimulation, samples were proceeded in biological triplicates (culture wells) and technical duplicates (for ELISA only), summarized by the mean of the values for each individual. Data are presented as means and standard error of the mean. Mann-Whitney tests were used to compare values between non-stimulated and stimulated purified T cells from HV. ${ }^{*} p<0.05,{ }^{* *} p<0.01$, ${ }^{* * *} p<0.001$. See Additional file 1 for details

Altogether, we report here an intrinsic downregulation of IL-7R soluble transcripts in septic shock patients' $T$ cells, independently of lymphopenia, in parallel with an increased sCD127 protein release. Ex vivo experiments in cells from HV suggest that initial $\mathrm{T}$ cell activation after sepsis might participate in this regulation, although this remains to be formally demonstrated.

\section{Additional file}

Additional file 1: Methods. (PDF $352 \mathrm{~kb}$ )

\section{Abbreviations}

HV: Healthy volunteer; IL-7R: IL-7 receptor; sCD127: Soluble CD127; aCD3/28: Anti-CD3/CD28 antibodies coated beads

\section{Acknowledgements}

The authors would like to thank Elisabeth Cerrato and Anne Portier for their help in experimental procedures, Olivier Tabone for his advices on statistical analysis and data representation, and Valérie Cerro and Justine Dubreuil for valuable help with the patient inclusion process in the Immunosepsis study.

\section{Funding}

This work was supported by bioMérieux (JM, EP, JT), Hospices Civils de Lyon (CA, TR, TU, BD, FV, GM), and Association Nationale de la Recherche et de la Technologie (JM, Convention Industrielle de Formation par la Recherche convention 2015/060). The Association Nationale de la Recherche et de la Technologie had no role in study design, in the collection, analysis, and interpretation of data and in writing the manuscript.

\section{Availability of data and materials}

The datasets used and/or analyzed during the current study are available from the corresponding author on reasonable request.

\section{Authors' contributions}

JM, CA, FV, and EP designed the experiments. JM and CA performed the experiments and the statistical analyses. All authors discussed the data, drafted or revised critically the manuscript for important intellectual content, and read and approved the final manuscript.

\section{Ethics approval and consent to participate}

Septic shock patients: this project was approved by our Institutional Review Board for ethics ("Comité de Protection des Personnes Sud-Est II"), which waived the need for informed consent, because this study was observational and 
performed on residual blood after completion of routine follow-up (\#IRB 11236). This study is registered at the French Ministry of Research and Teaching (\#DC-2008-509), at the Commission Nationale de I'Informatique et des Libertés and on clinicaltrials.gov (NCT02803346). Non-opposition to inclusion in the study was registered for each patient. Healthy volunteers: peripheral blood from healthy volunteers was provided by the "Etablissement Français du Sang" from Lyon. According to the standardized procedure for blood donation, written informed consent was obtained from healthy volunteers and personal data were anonymized at time of blood donation and before blood transfer to our research lab.

\section{Consent for publication}

Not applicable.

\section{Competing interests}

$J M, J T$, and EP are employees of bioMérieux. BD, FV, GM, EP, and JT are co-inventors on three patent families covering IL-7 receptor biomarkers. This work was supported by Association Nationale de la Recherche et de la Technologie (JM, Convention Industrielle de Formation par la Recherche convention 2015/060).

\section{Publisher's Note}

Springer Nature remains neutral with regard to jurisdictional claims in published maps and institutional affiliations.

\section{Author details}

${ }^{1}$ EA 7426 « Pathophysiology of injury-induced immunosuppression (PI3) » Lyon 1 University / Hospices Civils de Lyon / bioMérieux, 5 place d'Arsonval, 69437 Lyon Cedex 03, France. ${ }^{2} J$ oint Research Unit HCL-bioMérieux-Lyon 1 University, Hôpital Edouard Herriot, 5 place d'Arsonval, 69003 Lyon, France. ${ }^{3}$ Immunology Laboratory, Hospices Civils de Lyon, Hôpital Edouard Herriot, 5 place d'Arsonval, 69003 Lyon, France. ${ }^{4}$ Anesthesiology and Intensive care medicine department, Hospices Civils de Lyon, Hôpital Edouard Herriot, 5 place d'Arsonval, 69003 Lyon, France.

\section{Received: 7 August 2018 Accepted: 26 December 2018}

Published online: 08 January 2019

\section{References}

1. Hotchkiss RS, Coopersmith CM, McDunn JE, Ferguson TA (2009) Tilting toward immunosuppression. Nat Med 15:496497. https://doi.org/10.1038/nm0509-496

2. Francois B, Jeannet R, Daix T et al (2018) Interleukin-7 restores lymphocytes in septic shock: the IRIS-7 randomized clinical trial. JCl Insight 3. https://doi.org/10.1172/jci.insight.98960

3. Lundström W, Fewkes NM, Mackall CL (2012) IL-7 in human health and disease. Semin Immunol 24:218-224. https://doi. org/10.1016/j.smim.2012.02.005

4. Goodwin RG, Friend D, Ziegler SF et al (1990) Cloning of the human and murine interleukin-7 receptors: demonstration of a soluble form and homology to a new receptor superfamily. Cell 60:941-951. https://doi.org/10.1016/00928674(90)90342-C

5. Vranjkovic A, Crawley AM, Gee K et al (2007) IL-7 decreases IL-7 receptor a (CD127) expression and induces the shedding of CD127 by human CD8+ T cells. Int Immunol 19:1329-1339. https://doi.org/10.1093/intimm/dxm102

6. Rose T, Lambotte O, Pallier C et al (2009) Identification and biochemical characterization of human plasma soluble IL-7R: lower concentrations in HIV-1-infected patients. J Immunol 182:7389-7397. https://doi.org/10.4049/jimmunol.0900190

7. Peronnet E, Mouillaux J, Monneret $\mathrm{G}$ et al (2016) Elevated soluble IL-7 receptor concentration in non-survivor ICU patients. Intensive Care Med 42:1639-1640. https://doi.org/10.1007/s00134-016-4445-x

8. Delwarde B, Peronnet $\mathrm{E}$, Venet $\mathrm{F}$ et al (2018) Low interleukin-7 receptor messenger RNA expression is independently associated with day 28 mortality in septic shock patients. Crit Care Med 46:1739-1746. https://doi.org/10.1097/CCM. 0000000000003281

9. Alves NL, van Leeuwen EMM, Derks IAM, van Lier RAW (2008) Differential regulation of human IL-7 receptor a expression by IL-7 and TCR signaling. J Immunol 180:5201-5210. https://doi.org/10.4049/jimmunol.180.8.5201

10. Monti P, Brigatti C, Krasmann M et al (2013) Concentration and activity of the soluble form of the interleukin-7 receptor a in type 1 diabetes identifies an interplay between hyperglycemia and immune function. Diabetes 62:2500-2508. https://doi.org/10.2337/db12-1726

11. Schwulst SJ, Muenzer JT, Chang KC et al (2008) Lymphocyte phenotyping to distinguish septic from nonseptic critical illness. J Am Coll Surg 206:335-342. https://doi.org/10.1016/j.jamcollsurg.2007.07.038

12. Boomer JS, Shuherk-Shaffer J, Hotchkiss RS, Green JM (2012) A prospective analysis of lymphocyte phenotype and function over the course of acute sepsis. Crit Care 16:R112. https://doi.org/10.1186/cc11404 